\title{
TOWARDS IN VITRO VASCULARISATION OF COLLAGEN-GAG SCAFFOLDS
}

\author{
Garry P. Duffy ${ }^{1,2}$, Tara M. McFadden ${ }^{1,2}$, Elaine M. Byrne ${ }^{1,2}$, Sarah-Louise Gill ${ }^{1,3}$, Eric Farrell ${ }^{3,4}$ \\ and Fergal J. O’Brien ${ }^{1,2, *}$ \\ ${ }^{1}$ Department of Anatomy, Royal College of Surgeons in Ireland, Dublin 2, Ireland \\ ${ }^{2}$ Trinity Centre for Bioengineering, Trinity College, Dublin 2, Ireland \\ ${ }^{3}$ Regenerative Medicine Institute, National University of Ireland, Galway, Ireland \\ ${ }^{4}$ Department of Orthopaedics, Erasmus University Medical Centre, Rotterdam, The Netherlands
}

\begin{abstract}
Collagen-glycosaminoglycan scaffolds that have been used clinically for skin regeneration have also shown significant promise for other applications in tissue engineering. However, regeneration of thicker tissues with the aid of implanted biomaterials is likely to depend on, or be accelerated by, the ability to establish rapid vascularisation of the implant. The present study aims to establish a nascent vascular network in vitro within a CG scaffold as a first step towards that goal. Mesenchymal stem cells (MSCs) were chosen as primary vasculogenic candidate cells and a culture medium that promoted maximal network formation on Matrigel by these cells was selected. MSCs seeded in the CG scaffold formed networks of cord-like structures after one to two weeks in the presence of the vasculogenic medium; similar structures were formed by aortic endothelial cells (ECs) cultured for comparison. Gene expression analysis suggested that the MSCs began to adopt an endothelial phenotype, with RNA for PECAM and VCAM rising while that for $\alpha$-smooth muscle actin fell. However there was no increase in Tie-2 and vWF expression. Addition of smooth muscle cells (SMCs) as a potential perivascular stabilising component did not have a noticeable effect on MSC-derived networks, although it enhanced EC-derived structures.
\end{abstract}

Keywords: Tissue engineering, collagen-GAG scaffold, pre-vascularisation, mesenchymal stem cells, endothelial cells, smooth muscle cells, co-culture.

\footnotetext{
*Address for correspondence:

Fergal J. O’Brien,

Royal College of Surgeons in Ireland

123 St. Stephen's Green, Dublin 2

Ireland
}

Telephone Number: (01) 4022149

E-mail: fjobrien@rcsi.ie
CG: collagen-glycosaminoglycan

MSC: mesenchymal stem cell

EC: endothelial cell

SMC: smooth muscle cell

VEGF: vascular endothelial growth factor

EDAC: 1-ethyl-3-(3-dimethylaminopropyl)carbodiimide

NHS: N-hydroxysuccinimide

H\&E: haematoxylin and eosin

DMEM: Dulbecco's modified Eagle medium

PBS: phosphate-buffered saline

DAPI: 4',6-diamidino-2-phenylindole

vWF: von Willebrand factor

$\alpha$ SMA: alpha smooth muscle actin

bFGF: basic fibroblast growth factor

EGF: epidermal growth factor

PECAM: platelet endothelial cell adhesion molecule

\section{Introduction}

Proximity to a capillary network is essential for survival of most cell types, to enable delivery of oxygen, nutrients and systemic regulatory molecules and for removal of metabolic waste products. Thus, while tissue engineering aims to replace lost host tissues via constructs of biomaterial scaffolds and in vitro seeded tissue-specific cells, these implants generally cannot sustain viable cells in their interior during the time it takes for vessel ingrowth to occur from the host resulting in core degradation and necrosis (Ko et al., 2007; Rouwkema et al., 2008). Consequently, the limited clinical success of such work thus far has been restricted to the replacement of relatively thin or avascular tissues, which can rely on diffusion for nutrient supply e.g., skin and cartilage.

An FDA-approved type I collagen-glycosaminoglycan scaffold (Yannas et al., 1989; O'Brien et al., 2005) has been shown to support osteogenic activity in vitro (Farrell et al., 2006; Farrell et al., 2007; Byrne et al., 2008) and it is hoped to apply this scaffold to bone regeneration. More generally, a number of variants of this scaffold are being developed in our lab (Al-Munajjed and O'Brien, 2009; Al-Munajjed et al., 2009; Tierney et al., 2009a; Tierney et al., 2009b), with potential application to a wide variety of tissues, and it is envisaged that pre-vascularised constructs could support tissue-specific cells migrating from an implant site, or, alternatively, added in vitro.

Impaired angiogenesis plays a critical role in the occurrence of delayed healing and non-union fractures. 
Therefore it is strategically important to address angiogenesis when developing tissue engineered bone substitutes, not only to support the addition of tissuespecific cells in vitro but also to enhance the healing process in vivo. Bone formation and regeneration require a blood supply to be established during the early or later stages of the intramembranous or endochondral versions of the process, respectively. Models of bone tissue engineering by endochondral ossification (Jukes et al., 2008; Farrell et al., 2009) are thus an exception to the general principle that vessel ingrowth from the host would best be instant, but intramembranous models would benefit from rapid perfusion. The aim of the present study is to pre-vascularise the $\mathrm{CG}$ scaffold for use in the intramembranous model, i.e. to engineer capillary-like networks within this scaffold in vitro which might then join with host vasculature in vivo, in order to accelerate the process and minimise the chance of cell death in the interior of an implant. The scaffold is highly porous and the pores are well interconnected, so initial networks could form without need for any degradation of the scaffold.

Mesenchymal stem cells (MSCs), which have recently shown potential to differentiate towards the endothelial lineage (Oswald et al., 2004; Jazayeri et al., 2008), were employed here as the chief candidate vasculogenic cells as they are more accessible clinically than vascular endothelial cells. Aortic endothelial cells were also used in parallel in this study to more thoroughly test the vasculogenesis model for this scaffold. The addition of smooth muscle cells (SMCs) was also investigated as a potential stabilising element for the nascent vascular structures in the scaffold.

\section{Materials and Methods}

\section{CG scaffold}

CG scaffolds ( $95.5 \%$ porosity, $3 \mathrm{~mm}$ thick) were fabricated using a lyophilisation process as previously described (O'Brien et al., 2005) at a freezing temperature of $-40^{\circ} \mathrm{C}$ to generate an average pore size of $96 \mu \mathrm{m}$. Discs of 1.11 or $0.95 \mathrm{~cm}$ diameter $\left(0.97\right.$ or $0.71 \mathrm{~cm}^{2}$ area respectively) were cut using a biopsy punch and chemically crosslinked using 1-ethyl-3-(3-dimethylaminopropyl)carbodiimide (EDAC) in combination with N-hydroxysuccinimide (NHS) as described previously (Jaasma and O'Brien, 2008).

To illustrate the scaffold pore structure, a sample of scaffold was formalin-fixed, dehydrated, wax embedded and transverse sections of $10 \mu \mathrm{m}$ cut with a microtome; these were mounted on a glass slide, de-waxed, rehydrated, stained with haematoxylin and eosin (H\&E) and imaged with a ECLIPSE 90i (Nikon, Tokyo, Japan) microscope.

\section{Cell isolation and expansion}

All culture incubations were carried out at $37^{\circ} \mathrm{C}, 5 \% \mathrm{CO}_{2}$ and $95 \%$ relative humidity.

MSCs were isolated on the basis of plastic adherence from bone marrow of Wistar rat sacrificed by $\mathrm{CO}_{2}$ asphyxiation. The cells were expanded in Dulbecco's modified Eagle medium (DMEM, high-glucose SigmaAldrich (Dublin, Ireland) D5671 formulation) supplemented with $10 \%$ foetal bovine serum, $100 \mathrm{U} / \mathrm{ml}$ penicillin, $100 \mu \mathrm{g} / \mathrm{ml}$ streptomycin, $2 \mathrm{mM}$ Glutamax, 1 $\mathrm{mM}$ L-glutamine, and 1\% non-essential amino acids, essentially as described in (Farrell et al., 2006). Marrow was flushed from femoral and tibial shafts with culture medium using a 25-gauge needle. After pelleting by centrifugation for $5 \mathrm{~min}$ at $650 \mathrm{~g}$, it was passed sequentially through 16-, 18- and 20-gauge needles, then through a 40 $\mu \mathrm{m}$ cell strainer to remove clumps. This suspension was incubated in a $10 \mathrm{~cm}$ plastic Petri dish for a short time (30 $\mathrm{min}$ ) to deplete white blood cells and the supernatant was plated into two T75 tissue culture flasks. Cells that were non-adherent after $24 \mathrm{~h}$ were removed by rinsing and replacing medium in these flasks. Upon reaching 80-90\% confluence, the cells were passaged at a ratio of 1:2. Confluent flasks were rinsed with $10 \mathrm{ml}$ Dulbecco's phosphate buffered saline solution (DPBS) and $0.25 \%$ trypsin-EDTA solution (Sigma-Aldrich) was used to detach the cells from the surface of the flasks. Adherent proliferating cells were further passaged for several weeks to doubled flask numbers as they expanded, and half of the medium was replaced at least every 3-4 days if time between passages was longer. Cells from sets of 3 rats were pooled at passage 2 or 3 and used at passages 3-12.

Rat ECs and Rat Endothelial Cell Growth Medium (Cell Applications, Inc., San Diego, CA, USA; hereafter referred to as "EC medium") were purchased from ECACC (Salisbury, UK). The ECs were expanded in EC medium on gelatin-coated tissue-culture plastic and used at passage 7-10. Rat aortic SMCs (same source) were expanded initially in Rat Smooth Muscle Cell Growth Medium (same source) and then in low-glucose DMEM (Sigma-Aldrich, D6046 formulation, supplemented as for the high-glucose formulation, except that glutamine was already present), and used at passage 5 .

\section{Characterisation of rMSCs by flow cytometry}

The cell surface phenotype of rMSCs at passage 0 and passage 3 were characterized using flow cytometry analysis (Aria II, Becton Dickenson (BD), Franklin Lakes, NJ, USA). Briefly, cells were fixed using 5\% Formalin for 20 min. Cells were then centrifuged for $5 \mathrm{~min}$ at $500 \mathrm{xg}$ and resuspended in $200 \mu \mathrm{l}$ staining buffer (PBS and $1 \%$ Bovine Serum Albumin (BSA); Sigma-Aldrich). A 1 in 200 dilution of each antibody was added to $5 \times 10^{5}$ cells and left in the dark for $20 \mathrm{~min}$ at room temperature. Cells were then centrifuged as before and resuspended in wash buffer (PBS, 0.1\% TWEEN and 1\% BSA). Cells were then resuspended in $200 \mu \mathrm{l}$ PBS and filtered using a $50 \mu \mathrm{m}$ filter (Partec, Canterbury, UK). The following antibodies from $\mathrm{BD}$ Pharmingen ${ }^{\mathrm{TM}}$ (unless otherwise stated) were used: FITC anti-human CD45 (leukocyte common antigen, eBiosciences (Hatfield, UK)), FITC anti-rat CD71 (Transferrin Receptor, eBiosciences), Alexa Fluor ${ }^{\circledR} 488$ (Invitrogen, Carlsbad, CA, USA) anti- rat Tie-2, PE antirat CD106 (VCAM-1), FITC anti-rat CD73 (Ecto-5'nucleotidase), PE anti-rat CD31 (PECAM-1), FITC antiRat CD29 (Integrin $\beta 1$ ). Analysis was carried out using 
BD DIVA software. Histograms depict both positive cells and negative cells overlaid per test; the \% parent population expressing the antigen was also quantified and shown in the plot.

\section{Labelling cells with fluorescent dyes}

Red or green fluorescent cell labels (PKH26 and PKH2 respectively, Sigma-Aldrich) were used to facilitate morphological observations, particularly in the scaffold, and to distinguish between input cell types in co-cultures. Labelling was carried out according to the manufacturer's instructions, with the following modifications/details: per $4 \times 10^{6}$ cells, the initial wash (serum-free medium) was in $10 \mathrm{ml}$, labelling ( $4 \mu \mathrm{M}$ dye) was in $1 \mathrm{ml}$ for $5 \mathrm{~min}$ and terminated by mixing in an equal volume $(1 \mathrm{ml})$ of serum then $10 \mathrm{ml}$ complete ( $10 \%$ serum) medium; after pelleting, resuspending and transferring to a fresh tube, cells were washed again in $10 \mathrm{ml}$ and then at least $2 \mathrm{ml}$ complete medium, and final resuspension was in an appropriate volume of experimental medium for plating/seeding at densities indicated elsewhere.

\section{Matrigel assay}

Growth Factor Reduced Matrigel ${ }^{\mathrm{TM}}$ (BD Biosciences) was set in a 48 -well plate at $0.15 \mathrm{ml} /$ well (approx. $1 \mathrm{~cm}^{2}$ ). Cells were plated in triplicate at $3 \times 10^{6}$ per well in $0.5 \mathrm{ml}$ of test media. Vascular endothelial growth factor (VEGF, Cell Sciences, Canton, MA, USA), where used, was at $50 \mathrm{ng}$ / $\mathrm{ml}$.

\section{Culture in CG scaffolds}

Scaffold discs were seeded, in at least triplicate per group, in multiwell plates with $2.5 \times 10^{5} \mathrm{MSCs}$ or ECs per $\mathrm{cm}^{2}$ on both sides. To do this, the EDAC-crosslinked scaffolds were first equilibrated in medium (DMEM or EC medium as stated for individual experiments) and then excess liquid removed and cells applied at $3.33 \times 10^{6} / \mathrm{ml}$ in the same respective medium to one side of the scaffold and incubated for $30 \mathrm{~min}$. The scaffold was then turned over and the process repeated for the other side, followed by addition of culture medium and incubation for up to 28 days. For cocultures, SMCs were applied after 11 days at $0.833,1.67$, $3.33,6.67$ or $13.3 \times 10^{6} / \mathrm{ml}$. Medium $(2-5 \mathrm{ml})$ was changed 2-3 times weekly.

\section{Imaging}

After final imaging of live scaffold cultures, constructs were rinsed twice in phosphate-buffered saline (PBS), fixed for one hour in $2 \%$ buffered formalin (10\% stock, SigmaAldrich, diluted in PBS), rinsed twice in PBS, stained with 4',6-diamidino-2-phenylindole (DAPI) dihydrochloride (Sigma-Aldrich) for $20 \mathrm{~min}$, rinsed twice in PBS and stored in same at $4^{\circ} \mathrm{C}$ until imaging.

Matrigel cultures were imaged with a Leica (Wetzlar, Gemany) DMIL microscope (5x objective, DFC420 C digital camera). Scaffold constructs were imaged with the Leica (10x objective) and with a Zeiss (www.zeiss.com) Observer.Z1 microscope (20x objective, AxioCam HSm camera, with 1.6x optovar for added magnification in some instances).

\section{Gene expression analysis}

Relative RNA levels for genes of interest were measured by reverse transcription followed by quantitative real-time polymerase chain reaction as follows: Total RNA was extracted using the Qiagen (Hilden, Germany) RNeasy Mini Kit and RNase-Free DNase Set according to the manufacturers' instructions, disrupting scaffolds in 600 $\mu \mathrm{l}$ of kit lysis buffer using an electric homogeniser (rotor stator), with quantification by spectrophotometry. Reverse transcriptions $(20 \mu \mathrm{l})$ were performed on $400 \mathrm{ng}$ of total RNA using the QuantiTect Reverse Transcription Kit (Qiagen) according to the manufacturer's instructions. Real-time PCR reactions $(15 \mu \mathrm{l})$ were performed in triplicate on a 7500 Real-Time PCR System (Applied Biosystems, Foster City, CA, USA) using the QuantiTect SYBR Green PCR Kit (Qiagen). The following predesigned validated rat primer sets from Qiagen (QuantiTect Primer Assays) were used: Rn_Pecam_1_SG (PECAM), Rn_Vcam_1_SG (VCAM), Rn_KDR_2_SG (VEGFR2), Rn_Vwf_1_SG (von Willebrand factor), Rn_Tek_1_SG (Tie2), Rn_Acta2_1_SG ( $\alpha$-Smooth Muscle Actin), Rn_Eng_2_SG (Endoglin), Rn_Tfrc_2_SG (Transferrin Receptor), Rn_Rnr1_1_SG (rRNA). Relative quantities of target RNAs were calculated by the comparative delta-delta $\mathrm{C}_{\mathrm{t}}$ technique (base 2) with rRNA as endogenous reference. $0.4 \mathrm{ng}$ of cDNA was used in the rRNA reactions and $30 \mathrm{ng}$ for the other genes.

\section{Histological analysis of lumen formation within the CG scaffold}

To illustrate lumen formation within vascular-like structures formed after cell seeding on the CG scaffold a sample of scaffold was formalin-fixed, sectioned and stained with haematoxylin and eosin (H\&E) as described above.

Lumen formation was also demonstrated through fluorescent labelling of the wax embedded sections. Cells were labelled using green Alexa Fluor 488 phalloidin (Molecular Probes, Invitrogen, Ireland; www.invitrogen. com) to stain the cytoskeleton. A 1:400 dilution of phalloidin in PBS was used, with $0.1 \%$ Triton X-100 added to permeabilise the cells. Sections were washed three times with PBS before Vectashield (Vector Laboratories, Burlingame, CA, USA) mounting medium with DAPI was added to label the cell nuclei. Digital images of all stained sections were obtained using an imaging system (AnalySIS, Nikon, Tokyo, Japan) in conjunction with a microscope (Olympus IX51, Olympus, Tokyo, Japan).

\section{Statistical analysis}

The RNA experiment was performed in triplicate with an $\mathrm{n}=3$ for each PCR reaction and values are presented as mean \pm standard deviation. Groups were compared using two-way ANOVA with post hoc Bonferroni's tests, and differences at $p<0.05$ were considered significant. 


\section{Results}

\section{Cell surface phenotype of MSCs}

Flow cytometry analysis demonstrated that rMSCs at the end of passage 0 were a heterogenous population and that the cells became more homogenous by the end of passage 3. Passage 0 cells had sub-populations positive for both EC (TIE-2, CD31, VCAM) and hematopoietic cell markers (CD45) whereas by P3 cells expressed only MSC related markers.

\section{Optimal medium for vasculogenic culture}

The ability of rat MSCs to form networks of cord-like structures on Matrigel was examined in the presence of a number of different culture media (Fig. 2). Compared to a low basal level of structure formation in standard MSC expansion medium, addition of VEGF in the presence of a lower serum concentration resulted in distinct network formations. However, the most extensive networks developed in EC medium, with or without VEGF, and these were similar to networks generated from rat aortic endothelial cells in the same medium (Fig. 2, inset). EC medium was therefore chosen as the best candidate medium for vasculogenesis with either cell type in the CG scaffold.

\section{MSCs and ECs can form network-like structures in the CG scaffold}

CG scaffolds (Fig. 3A) were then seeded with MSCs and cultured in EC medium for 28 days. MSCs formed network-like structures in the scaffold within approximately 10 days and these persisted for up to four weeks, suggestive of vasculogenic potential (Fig. 3B). To further characterize these structures, MSC cultures were compared to EC cultures over the same time period (Fig. 4). The morphology displayed by the MSC populations (left) in the scaffold was similar to that for the ECs (right) at all time points throughout the assay, suggesting that the MSCs are adopting an endothelial-like behaviour in this three-dimensional substrate.

In contrast, distinct multicellular structures did not develop in MSC-seeded scaffolds cultured in MSC expansion medium (high-serum DMEM; Fig. 4B). Strikingly, these constructs underwent marked cellmediated contraction (Fig. 4, column I), while there was little contraction with either cell type in EC medium (Fig. 4, columns II and III).

\section{Gene expression profile of endothelial markers in MSCs on the CG scaffold under angiogenic conditions}

Relative levels of a number of angiogenesis- and stem cellassociated RNAs were evaluated in MSC-seeded constructs cultured in EC medium, and, for comparison, the non-angiogenic DMEM, over twenty four days (Fig. 5). Expression was also measured in ECs at the final time point (Fig. 5, insets). PECAM and VCAM increased in the MSCs during the course of culture in the presence of EC medium and were significantly elevated over the level in DMEM-cultured constructs at the final time point, although not reaching the levels seen in ECs. Three other angiogenesis-related markers, von Willebrand factor (vWF), VEGF-R2 and Tie-2, however, remained low. RNA for alpha smooth muscle actin ( $\alpha$ SMA) fell significantly farther in the presence of EC medium than DMEM, while levels in the ECs were lower still. Endoglin (CD105) RNA also fell for the MSCs in EC medium and was significantly lower at the final time point than in DMEM. A similar trend was seen for Transferrin Receptor (or CD73) RNA, another marker of undifferentiated MSCs, which fell to a level equal to that in ECs at day 24 of scaffold culture in EC medium.

\section{Addition of SMCs enhances EC networks in the CG scaffold}

In order to test the hypothesis that SMCs would enhance the structure or stability of MSC and EC networks in the CG scaffold, constructs containing PKH26-labelled MSCs or ECs were cultured in EC medium for eleven days to allow initial network formation and then PKH2-labelled aortic SMCs were seeded at one quarter, one half, equal, two times or four times the density of the MSCs/ECs, i.e. at MSC/EC:SMC ratios of 1:4, 1:2, 1:1, 2:1, 4:1. The coculture constructs, along with MSC- or EC-only controls, were then incubated for an additional fourteen days and examined at intervals by microscopy. There were no appreciable differences in outcome among the six groups for the MSC networks (Fig. 6, 1:4 ratio not shown). For the EC networks, the presence of SMCs at the $4: 1$ and possibly 2:1 ratios appeared to result in larger, more defined structures (Fig. 7, 1:4 ratio not shown). Fig. 8 shows selected MSC and EC structures at SMC ratios up to $1: 1$ in more detail; the vessel-like structures seen in the micrographs for the 1:2 and 1:4 ratios of SMCs:ECs are several cells thick, containing both cell types suggesting that lower ratios enhance vascular structure formation.

\section{Formation of luminal structures in the EC/SMC co- cultures}

To assess whether there were lumens present within the vascular structures formed on the CG scaffold we carried out histology and immunofluorescent imaging on sections from day 28 samples. Lumens were observed within structures in all culture groups but there were a larger number present in the EC/SMC co-culture group (Fig. 9).

\section{Discussion}

Vascularisation is crucial to support cell loaded tissue engineered scaffolds and also enhance healing in bone defects. The goal of the present study was to establish a nascent capillary network in a well-characterised collagenGAG scaffold to support use of that scaffold in tissue engineering, primarily for bone regeneration. The most basic component of a capillary network is a tube of ECs. However vascular ECs are not an easily accessible resource for eventual clinical application, as they are not available in the necessary quantities to apply to tissue engineered scaffolds. MSCs are attractive as an easily accessible 

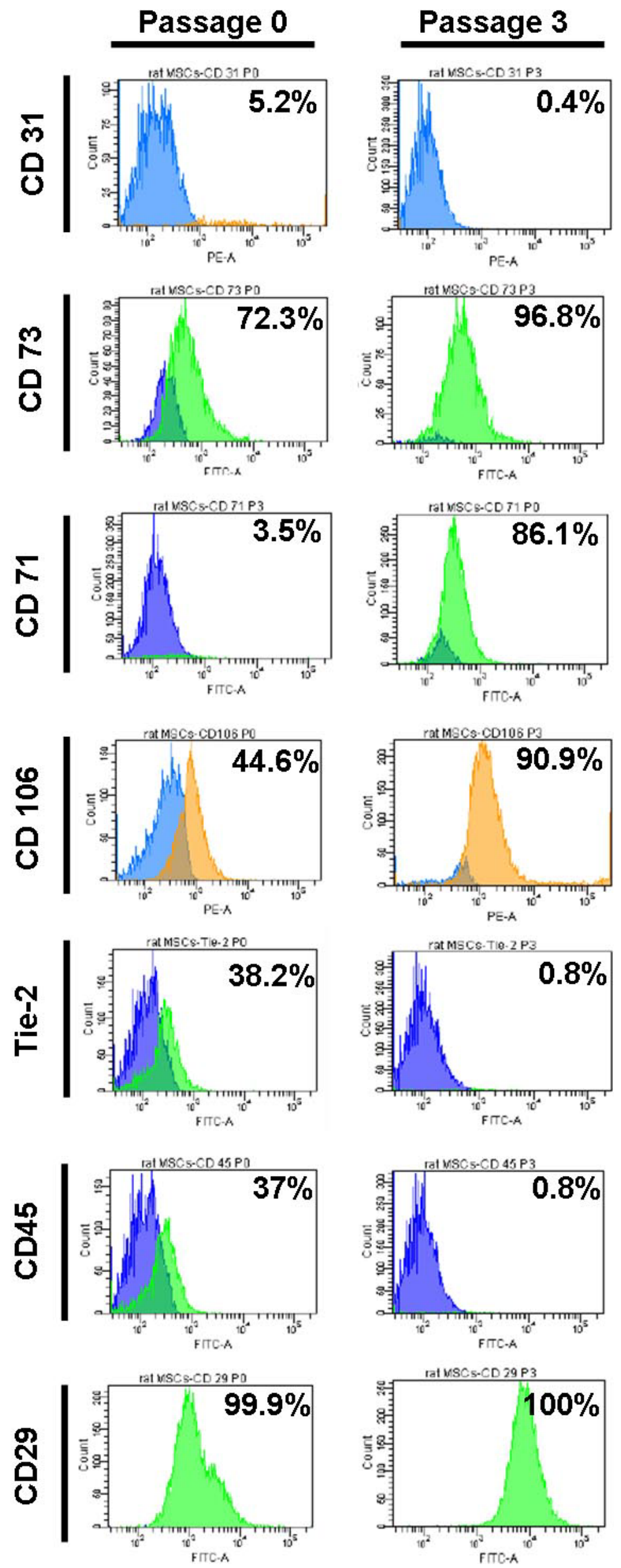

Fig. 1. Flow cytometry histogram plots depicting the cell surface phenotype of Passage 0 and Passage 3 cells. The number of positive cells (\% parent population) are included in each plot. Sub-populations of ECs (CD31, Tie-2, VECAM) and HSCs (CD45) are present in the P0 rMSCs but absent in P3 MSCs. 

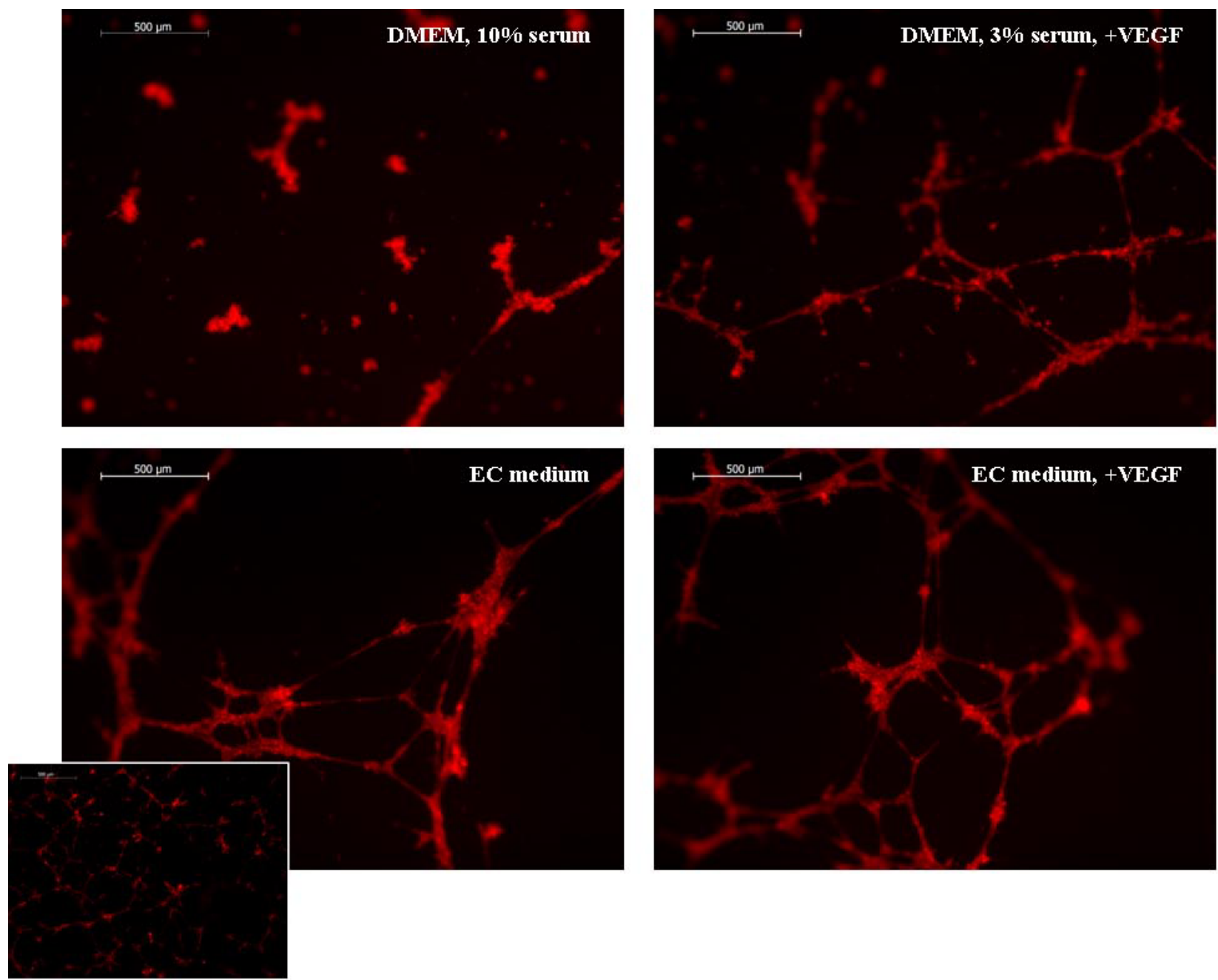

Fig. 2. Micrographs of PKH26-labelled MSCs after 12 hours on Matrigel in different media. Inset: ECs in EC medium after 11 hours for comparison. Network formation is strongest in EC medium.

A

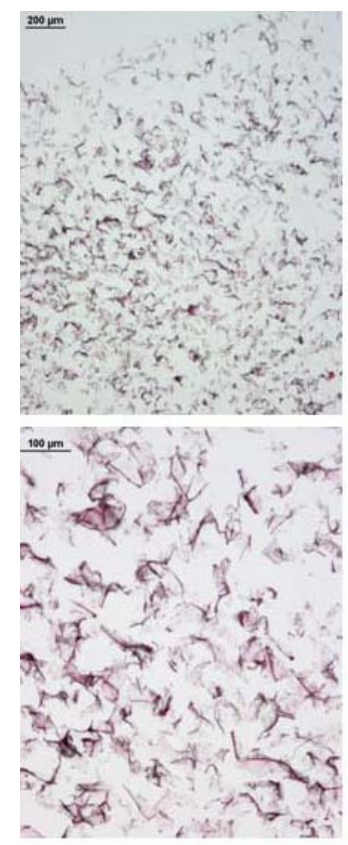

B

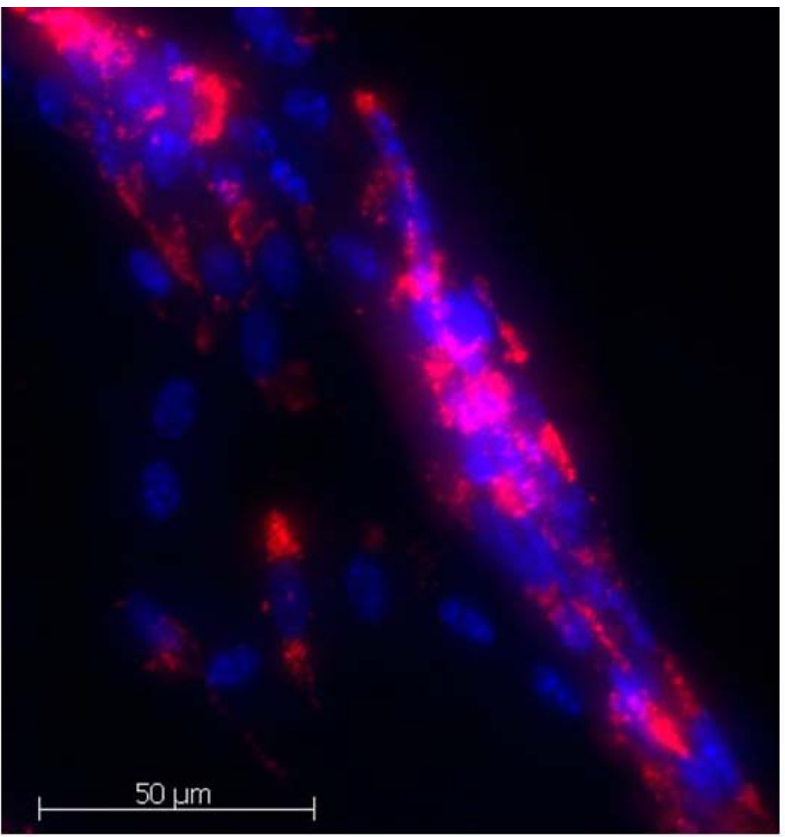

Fig. 3. Micrograph of H\&E-stained $10 \mu \mathrm{m}$ section of wax-embedded unseeded CG scaffold (A, upper), and at higher magnification (A, lower) showing the porous structure of the CG scaffold. Maximum projection from a Zstack of 30 images taken $1.23 \mu \mathrm{m}$ apart near the surface of a CG scaffold seeded with PKH26-labelled MSCs and cultured for 28 days in EC medium followed by formalin fixation and DAPI staining (B); cells are aligned into a cord-like structure. 
$\mathbf{A}$

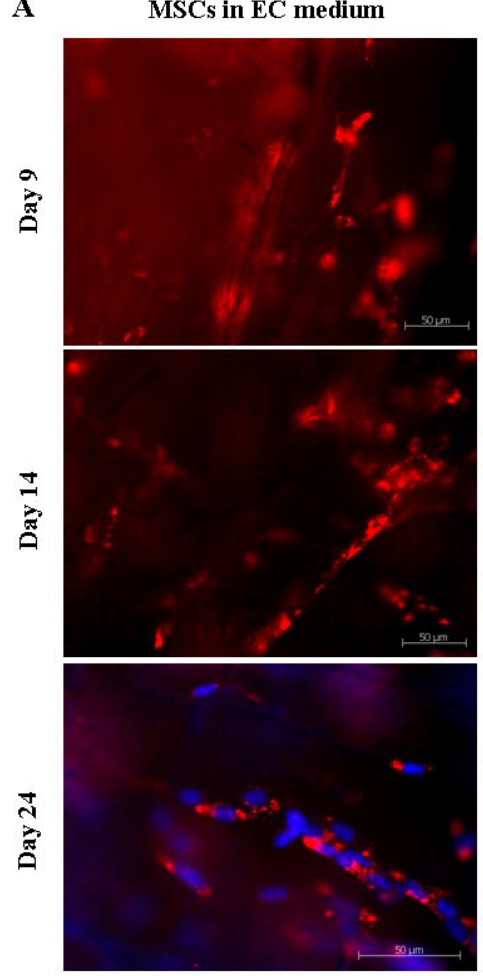

B MSCs in DMEM

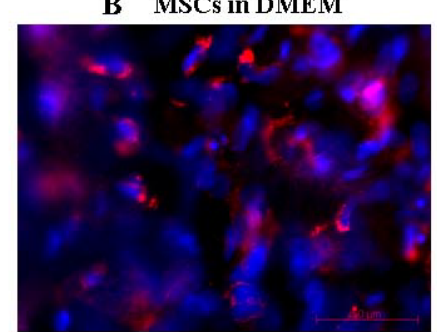

ECs in EC medium
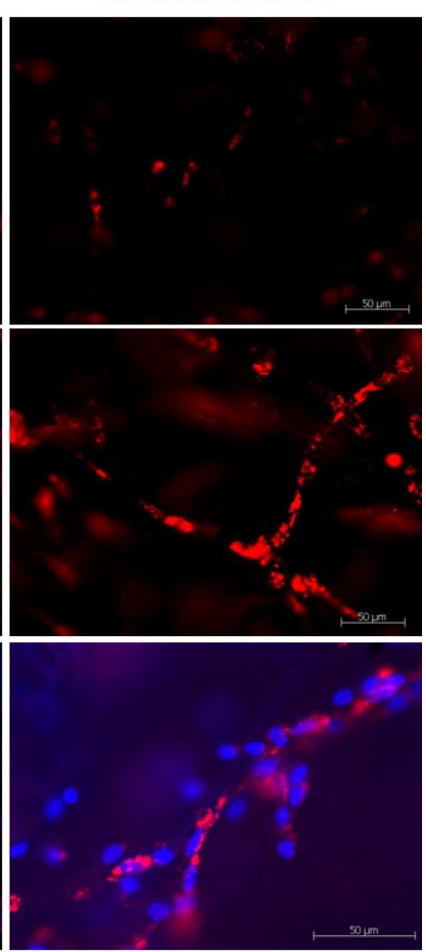

C

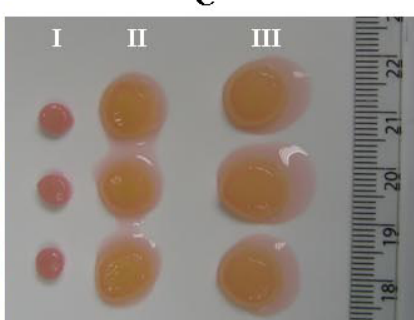

Fig. 4. Micrographs (Zeiss Observer) from nearsurface of CG scaffolds seeded with PKH26labelled MSCs (left) or ECs (right) in EC medium, with day 24 images taken after formalin fixation and DAPI staining at 1.6x higher magnification (A); networks of cord-like structures develop from both cell types. Similar structures are not seen in MSC-seeded scaffolds cultured in DMEM (B; 28 days, fixed and DAPI stained). Macroscopic photograph of day 24 constructs similar to those of (A) and (B) and used to provide RNA for Fig. 4 (C): MSCs in DMEM (column I), MSCs in EC medium (column II), ECs in EC medium (column III), ruler showing $\mathrm{cm}$ (C); scaffold discs of 1.11 $\mathrm{cm}$ diameter were used and it can be seen that those in column I underwent much greater contraction than those in the columns II and II. progenitor to many cell types and have recently shown potential to differentiate towards the endothelial lineage (Oswald et al., 2004; Jazayeri et al., 2008); these cells were therefore employed here as the chief candidate vasculogenic cells to test their ability to vascularise the CG scaffold in comparison with mature endothelial cells.

Melero-Martin et al. (2007, 2008) and Au et al. (2008) have demonstrated the feasibility of generating threedimensional host-connected capillary networks from cells implanted in vivo within Matrigel and collagen/fibronectin gels respectively. We sought to produce similar candidate implantation constructs with a CG scaffold. Compared to the above hydrogels, the material of the scaffold has a relatively coarser and stiffer architecture, which makes it more suitable as a substrate for clinical application. However these characteristics also mean that cells seeded for vascular purposes, on attaching to the struts, are less free to move and form networks than within the fine protein mesh of a hydrogel. Also, MSCs need to undergo major biochemical as well as morphological changes to initiate vasculogenesis. For these reasons, it was decided to observe the morphology and gene expression of the cells during extended in vitro pre-culture in the current study.

A two-dimensional Matrigel assay was first used to choose a medium most likely to induce MSCs to differentiate towards the endothelial phenotype and form pre-vascular networks in the CG scaffold. Candidate vasculogenic media included a low-serum version of the MSC expansion medium with the addition of the angiogenic/vasculogenic VEGF and an EC culture medium that contains other factors, plus or minus VEGF. EC medium was found to be more effective than low-serum DMEM plus VEGF, and the addition of VEGF to the EC medium did not appear to further enhance network formation. The results presented here are consistent with those of Oswald et al. (2004), who show fine networks on a Matrigel-like extracellular matrix gel in the presence of low serum DMEM with VEGF after two hours - we observed similar formations at this stage (not shown) from which the larger structures seen in Fig. 2 developed. Similarly, Alviano et al. (2007) saw that amniotic membrane-derived MSCs were stimulated by low serum medium with VEGF to form progressive structures over twenty hours and Cipriani et al. (2007) show similar results for bone marrow MSCs. The EC medium formulation (which also has low serum) contains basic fibroblast growth factor (bFGF) and epidermal growth factor (EGF) as well as heparin and hydrocortisone. bFGF has been used as an alternative to or with VEGF to stimulate vasculogenesis by ECs (Engelse et al., 2007; Finkenzeller 

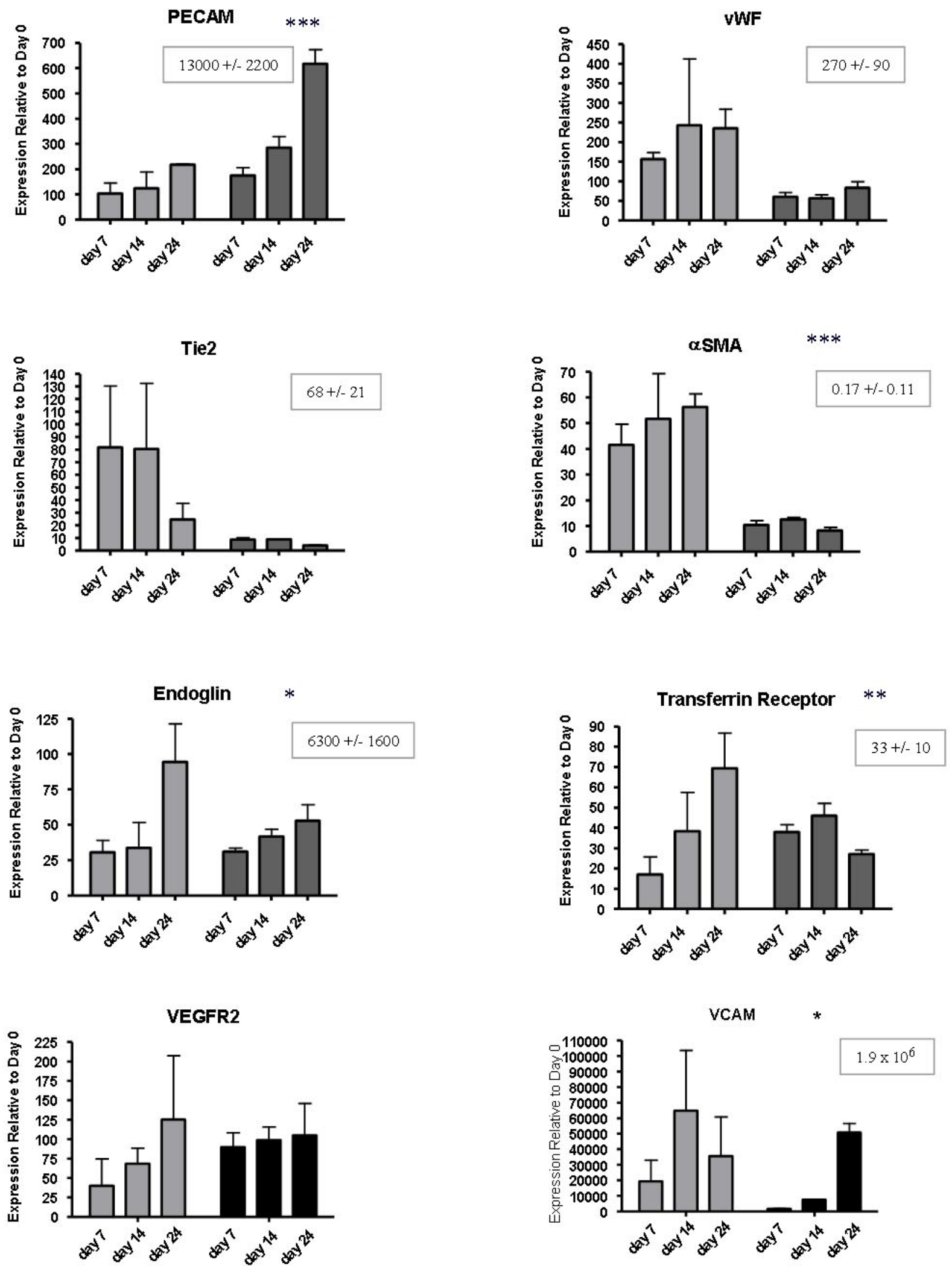

Fig. 5. Levels of RNAs for 8 differentiation-associated markers in DMEM- versus EC medium- cultured scaffold constructs as a percentage of the level in input MSCs at time of seeding; mean $+\mathrm{SD}, \mathrm{n}=3 ; * / * * / * * *$ represent $p<0.05 /$ 0.01/0.001 for differences in mean levels between DMEM and EC medium at day 24. Insets: Levels from ECs cultured for 24 days in EC medium in scaffold on same relative scale, shown to 2 significant figures, +/- SD. The rising PECAM and VCAM and falling $\alpha$ smooth muscle actin (SMA) for MSCs in EC medium suggest differentiation towards the endothelial phenotype, however no increases were seen for TIE-2, vWF and VEGFR2 suggesting this process did not follow through to completion. 

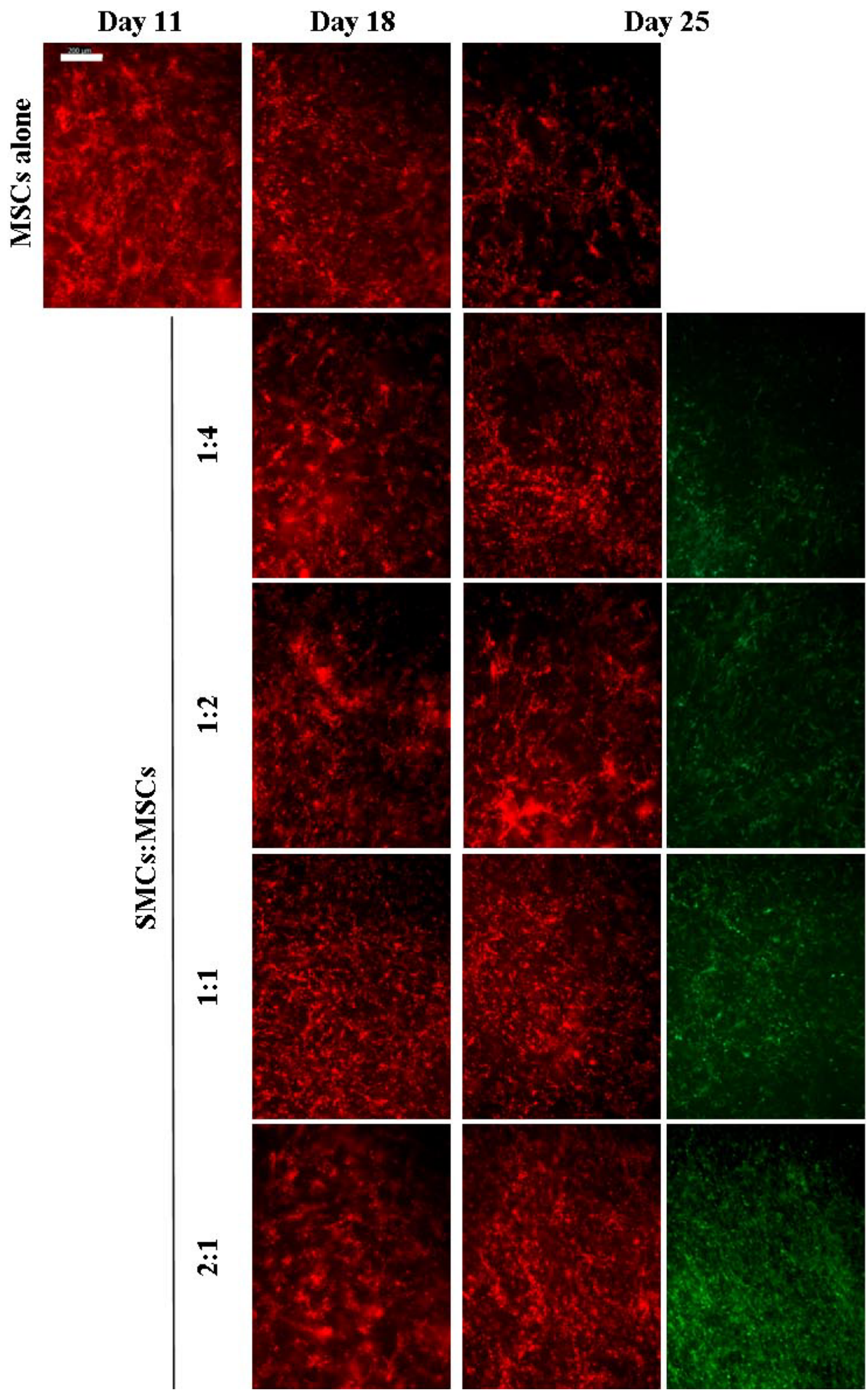

Fig. 6. Micrographs (Leica DMIL) from near-surface of CG scaffolds seeded with PKH26-labelled MSCs, cultured in EC medium and then seeded with PKH2-labelled SMCs after 11 days at the indicated ratios, then cultured for a further 14 days. Right column of day 25 shows SMCs (green channel). The SMCs have little or no effect on the MSC structures. Scale Bar represents $200 \mu \mathrm{m}$. 


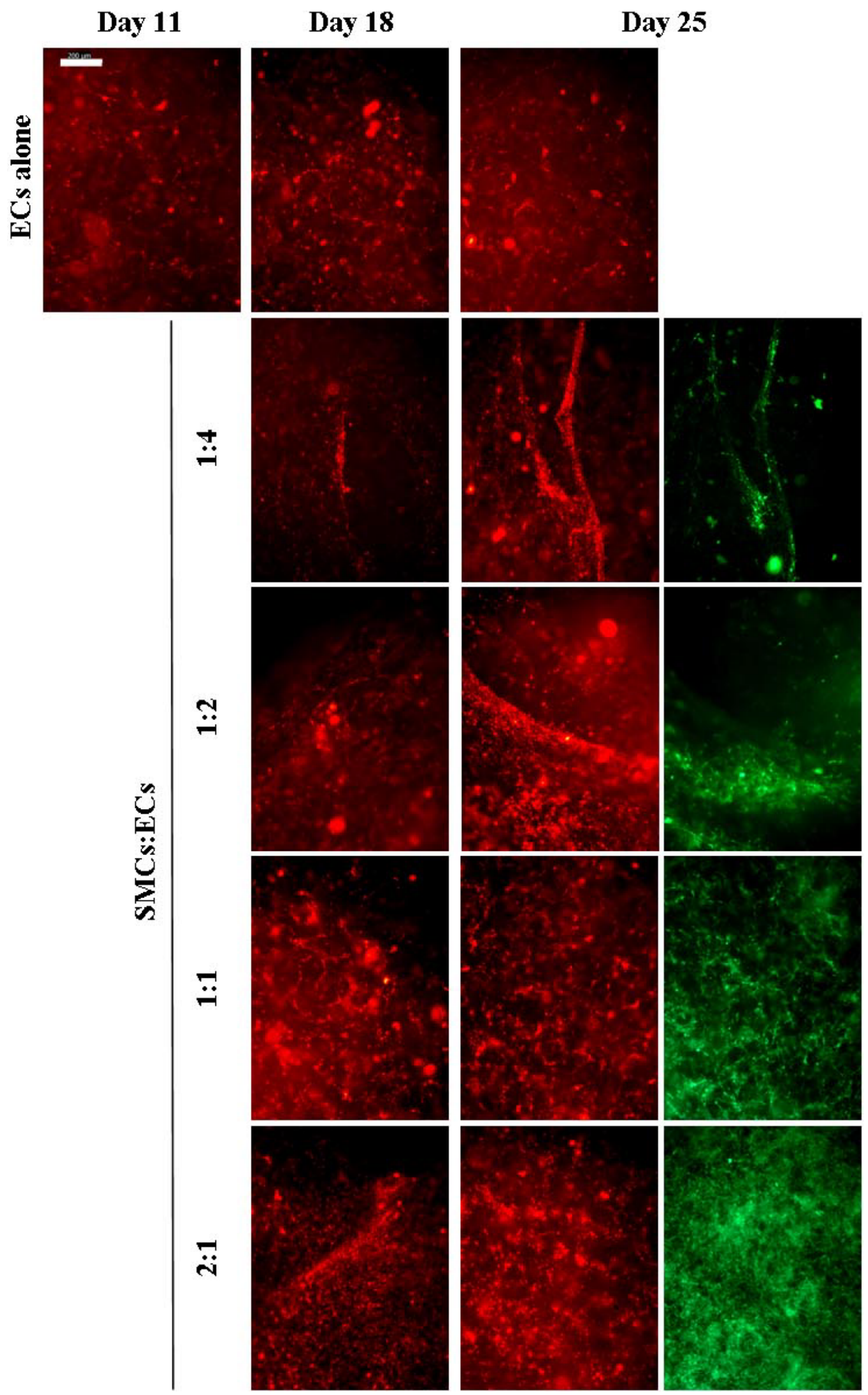

Fig. 7. Micrographs (Leica DMIL) from near-surface of CG scaffolds seeded with PKH26-labelled ECs, cultured in EC medium and then seeded with PKH2-labelled SMCs after 11 days at the indicated ratios, then cultured for a further 14 days. Right column of day 25 shows SMCs (green channel). The presence of SMCs at lower doses (1:4 and 1:2) appears to result in enhanced MSC structures. Scale bar represents $200 \mu \mathrm{m}$ 


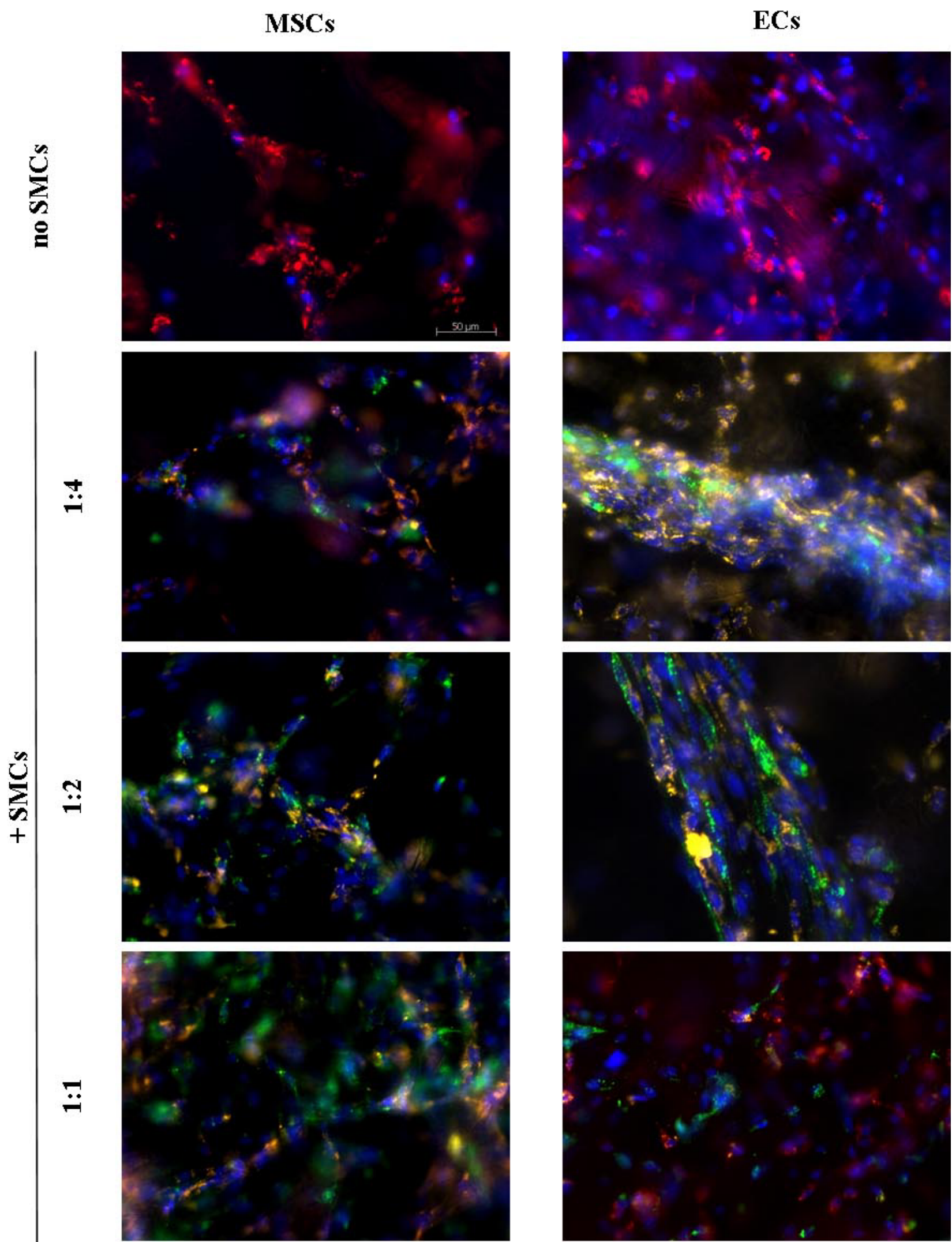

Fig. 8. Higher magnification images of selected day 25 constructs from the experiments portrayed in Figures 5 and 6 after fixing and DAPI-staining. Red or orange, MSCs (left column) or ECs (right column); green, SMCs; blue, DAPI-stained nuclei. Some structures in the co-cultures contain both MSCs and SMCs, with notably large cords seen in the 1:4 and 1:2 photographs of EC-SMC cocultures. 




ECs Alone
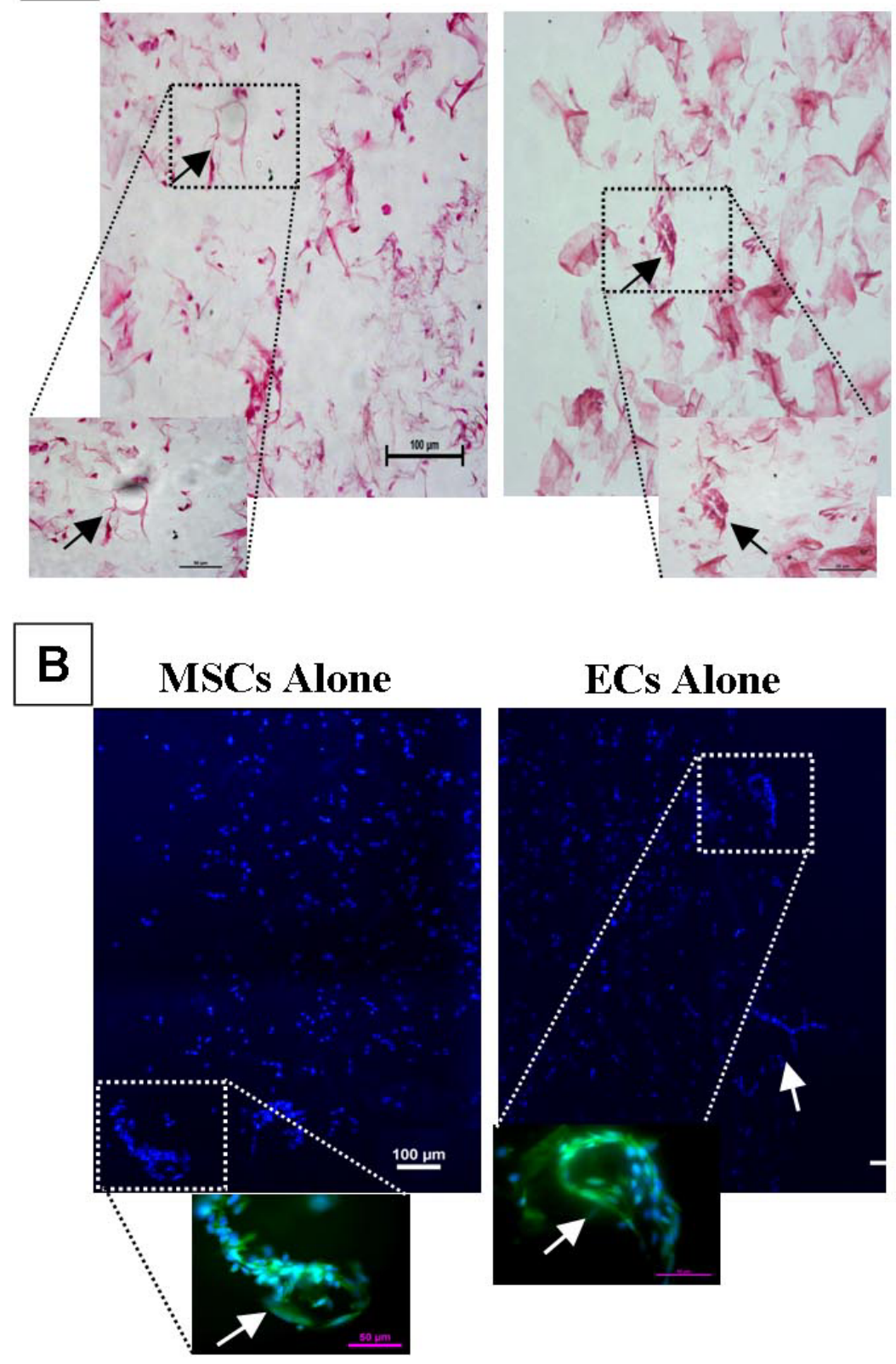

\section{ECs SMCs 2:1}

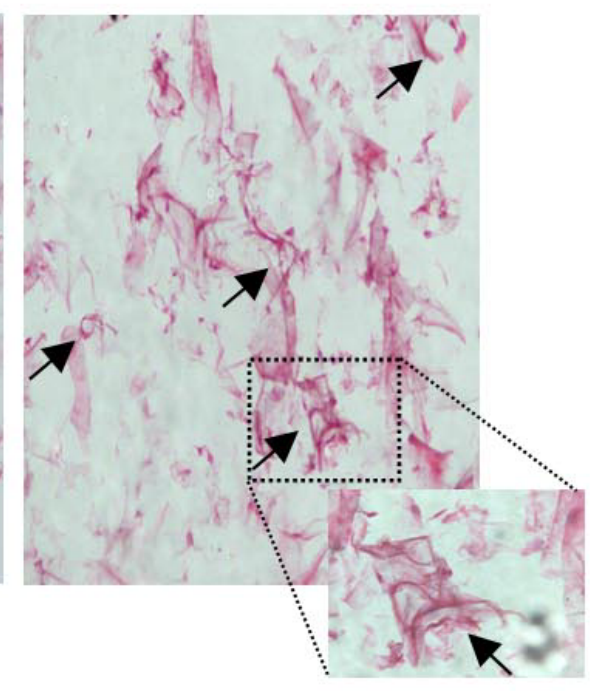

ECs SMCs 2:1

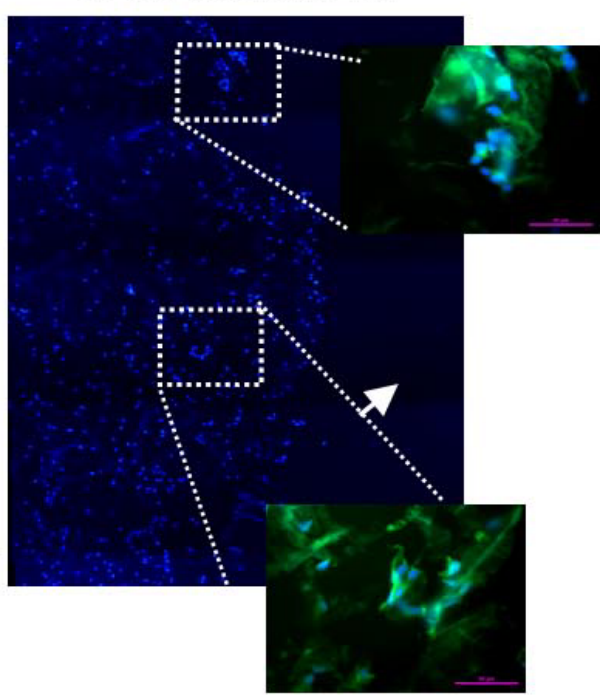

Fig. 9. Micrographs of H\&E-stained (A) and fluorescently-labelled (B) (Green, cytoskeleton and blue, DAPI-stained nuclei) $10 \mu \mathrm{m}$ sections of wax-embedded MSC, EC \& EC/SMC seeded CG scaffolds (Top panel: 20x), and at higher magnification (Bottom panel: 40x) showing the formation of vessel-like structures with lumens present. There were more vessel-like structures present in the EC/SMC co-culture. Scale bar represents $100 \mu \mathrm{m}$.

et al., 2007; Yamamura et al., 2007). It is likely that this factor is the main vasculogenic component in our EC medium, and it has been found to itself induce VEGF expression in ECs (Seghezzi et al., 1998); if the same is true in MSCs, or the MSCs are directly stimulated towards an EC phenotype first by the bFGF, this may be why additional VEGF did not promote further vascular structure formation by MSCs.

In the CG scaffold, MSCs and ECs formed networks of cord-like structures in the presence of the EC medium over the course of several days (Figs. 3B, 4A, 6 and 8). The absence of such self-organisation for MSCs in the presence of MSC expansion medium (high serum DMEM,
Fig. 3B) parallels the results of the Matrigel experiment, supporting the choice of EC medium for vasculogenic scaffold culture. On a two-dimensional film of CG, we noted that MSCs also display some movement, aggregation and changes in morphology in the presence of EC medium compared to a more uniform cell spreading in DMEM (data not shown), but the formations in the scaffold are much more striking. As the pores of the CG scaffold are interconnected, the scaffold itself is essentially a threedimensional network of cord-like struts and areas of more planar material (O’Brien et al., 2004). It would not be surprising if this structure influences that of the nascent cell networks: the arrangement of cells in the scaffold will 
at least initially be dictated by direct binding to the substrate, and those binding to the struts are likely to adopt linear formations. In this context, it is interesting to note that there are many reports of substrate adherence influencing cell phenotype, including work that showed tubule formation by ECs constrained to moderate spreading on linear adherent patches of approx. $10 \mu \mathrm{m}$ (Dike et al., 1999; Ingber, 2002).

Gene expression analysis was not consistent with the formation of vessel-like structures observed within the scaffold (Fig. 5). Two of the angiogenic markers assayed, PECAM and VCAM, rose as culture progressed in the EC medium, although vWF, VEGFR2 and Tie2 were not observed to increase. RNA for $\alpha$ SMA, on the other hand, quickly fell in the scaffold-cultured cells, to much lower levels in the presence of EC medium than DMEM, and was lower still in mature ECs, consistent with the MSCs differentiating to a cell type other than SMCs. $\alpha$ SMA is associated with a contractile phenotype, and the higher levels in DMEM-cultured MSC constructs is consistent with the marked cell-mediated contraction seen for these constructs, compared to the EC-cultured MSC and EC constructs. Falls in the endoglin and transferrin receptor RNAs are also consistent with MSC differentiation. These results suggest that EC medium induces MSCs in the CG scaffold to begin to differentiate towards the endothelial phenotype in vitro; however, this process did not proceed to completion. This may be due to a limitation of in vitro culture which does not provide the full physiological conditions required for vasculogenesis and angiogenesis i.e. blood flow and shear stress (Lee and Niklason, 2010).

Other groups report in vitro differentiation that may be more advanced. Oswald et al. (2004) observed expression of vWF after 7 days exposure to VEGF on plastic. In similar conditions, Alviano et al. (2007) also demonstrated VEGF-dependent vWF, as well as PECAM. Jazayeri et al. (2008) found that treating MSCs for five days with VEGF and IGF-1 in culture flasks induced expression of vWF RNA, PECAM, Tie 2 and $v W F$ proteins and changes in intracellular morphology consistent with an endothelial phenotype. Curiously, the induced cells then took a relatively long time (seven days) to form networks on an extracellular matrix extract similar to Matrigel. vWF staining has also been demonstrated by a group who found that mixed esters of butyric and retinoic acid induced differentiation (Ventura et al., 2007). There may be scope for employing additional factors such as these (IGF-1 and the mixed ester) into our protocol in order to further develop the MSC-derived nascent vasculature in our CG scaffold in vitro.

A perivascular layer of SMCs or pericytes surrounds, respectively, the larger and smaller endothelial tubes of the vasculature; in addition to having mechanical functions, the perivascular cells stabilise the endothelial layer in a viable but non-proliferating state with relatively tight intercellular connections (Carmeliet, 2000). Hence, interference with pericyte recruitment during vascular development in vivo results in regression of nascent endothelial vessels (Benjamin et al., 1998), while loss of pericyte coverage results in capillary leakage in diabetic retinopathy (Simo et al., 2006). MSCs themselves have been shown to play a role as a perivascular cell type in vivo (Caplan, 2008; Crisan et al., 2008; Melero-Martin et al., 2008). Consistent with this role, they have been shown to have pro-angiogenic effects on ECs in vitro (Hung et al., 2007). SMCs and MSCs have been demonstrated to stabilise EC networks on Matrigel in vitro, in a dosedependent fashion, if added within a short time after the network structures have formed (Duffy et al., 2009). In the implanted hydrogels discussed above (Au et al., 2008; Melero-Martin et al., 2008; Melero-Martin et al., 2007) SMCs or MSCs were used in the role of perivascular support for ECs.

Addition of SMCs was investigated here as a potential perivascular stabilising element for MSC- or EC-derived nascent vascular structures in the scaffold (Figs. 6-8). The SMCs were seeded at a range of densities, as ratio to endothelial component has been found important in studies quoted above (Duffy et al., 2009; Melero-Martin et al., 2008). Adding the SMCs resulted in the formation of larger structures in the EC-seeded scaffolds (Figs. 7 and 8) although the SMCs may associate with the cord-like structures produced by both the MSCs and ECs (Fig. 8). There are a number of possible reasons for the difference in response to the SMCs: (1) From the results obtained it seemed that the EC networks may have formed more slowly than the MSC structures, which were probably fully formed by day 11; in that case, SMCs might have had a greater influence over the formation of the EC structures or might have been able to stabilise otherwise transient formations for these cells. (2) It is possible that a minority of the MSCs may have retained their original phenotype in the MSC-seeded constructs and taken a role as pericytes around the majority endothelial-differentiating cells, before addition of the SMCs, which were then rendered functionally redundant. This may indicate that while the MSCs may not be the optimal cell type for inducing vasculogenesis within a $C G$ scaffold they may have potential to be used as a perivascular cell type for stabilizing any vessel formation in vitro.

The results presented here show that MSCs appear to form structures suggestive of early vascular formations in the CG scaffold when cultured in optimal medium in vitro. However analysis of gene expression indicates that while early EC differentiation may occur initially the process is not sustained and thus critical markers such as Tie- 2 and vWF are not expressed. ECs were also shown to form elaborate vascular-like structures within the CG scaffold and these may be the pro-angiogenic cell source of choice. These structures also appeared to be stabilized by the incorporation of a perivascular component in the form of SMCs and lumens were noted after histological examination.

Based on these results future work may focus on using MSCs as the perivascular component alongside an endothelial cell source to produce a fully vascularised CG scaffold. The use of endothelial progenitor cells may also be explored as the vasculogenic cell source as they are easier to isolate than fully differentiated ECs. Ultimately however, the optimal culture conditions required to 
vascularise the collagen-GAG scaffold will require in vivo assessment with the goal of achieving rapid anastomosis with host vasculature and formation of mature capillaries.

\section{Acknowledgements}

Funding for this study was provided by Science Foundation Ireland under the Research Frontiers Programme (27/ ENMF487) and President of Ireland Young Researcher Award (04/Y11/B531). Collagen materials were provided by Integra Life Sciences, Inc., through a Materials Transfer Agreement.

\section{References}

Al-Munajjed AA, O'Brien FJ (2009) Influence of a novel calcium-phosphate coating on the mechanical properties of highly porous collagen scaffolds for bone repair. J Mech Behav Biomed Mater 2: 138-146.

Al-Munajjed AA, Plunkett NA, Gleeson JP, Weber T, Jungreuthmayer C, Levingstone T, Hammer J, O'Brien FJ (2009) Development of a biomimetic collagenhydroxyapatite scaffold for bone tissue engineering using a SBF immersion technique. J Biomed Mater Res B Appl Biomater 90: 584-591.

Alviano F, Fossati V, Marchionni C, Arpinati M, Bonsi L, Franchina M, Lanzoni G, Cantoni S, Cavallini C, Bianchi F, Tazzari PL, Pasquinelli G, Foroni L, Ventura C, Grossi A, Bagnara GP (2007) Term Amniotic membrane is a high throughput source for multipotent Mesenchymal Stem Cells with the ability to differentiate into endothelial cells in vitro. BMC Dev Biol 7: 11.

Au P, Tam J, Fukumura D, Jain RK (2008) Bone marrow-derived mesenchymal stem cells facilitate engineering of long-lasting functional vasculature. Blood 111: 4551-4558.

Benjamin LE, Hemo I, Keshet E (1998) A plasticity window for blood vessel remodelling is defined by pericyte coverage of the preformed endothelial network and is regulated by PDGF-B and VEGF. Development 125: 15911598.

Byrne EM, Farrell E, McMahon LA, Haugh MG, O'Brien FJ, Campbell VA, Prendergast PJ, O'Connell BC. (2008) Gene expression by marrow stromal cells in a porous collagen-glycosaminoglycan scaffold is affected by pore size and mechanical stimulation. J Mater Sci Mater Med 19: 3455-3463.

Caplan AI (2008) All MSCs are pericytes? Cell Stem Cell 3: 229-230.

Carmeliet P (2000) Mechanisms of angiogenesis and arteriogenesis. Nat Med 6: 389-395.

Cipriani P, Guiducci S, Miniati I, Cinelli M, Urbani S, Marrelli A, Dolo V, Pavan A, Saccardi R, Tyndall A, Giacomelli R, Cerinic MM (2007) Impairment of endothelial cell differentiation from bone marrow-derived mesenchymal stem cells: new insight into the pathogenesis of systemic sclerosis. Arthritis Rheum 56: 1994-2004.

Crisan M, Yap S, Casteilla L, Chen CW, Corselli M, Park TS, Andriolo G, Sun B, Zheng B, Zhang L, Norotte C, Teng PN, Traas J, Schugar R, Deasy BM, Badylak S,
Buhring HJ, Giacobino JP, Lazzari L, Huard J, Peault B (2008) A perivascular origin for mesenchymal stem cells in multiple human organs. Cell Stem Cell 3: 301-313.

Dike LE, Chen CS, Mrksich M, Tien J, Whitesides GM, Ingber DE (1999) Geometric control of switching between growth, apoptosis, and differentiation during angiogenesis using micropatterned substrates. In Vitro Cell Dev Biol Anim 35: 441-448.

Duffy GP, Ahsan T, O’Brien T, Barry F, Nerem RM (2009) Bone marrow-derived mesenchymal stem cells promote angiogenic processes in a time- and dosedependent manner in vitro. Tissue Eng Part A 15: 24592470.

Engelse MA, Laurens N, Verloop RE, Koolwijk P, van Hinsbergh VW (2008) Differential gene expression analysis of tubule forming and non-tubule forming endothelial cells: CDC42GAP as a counter-regulator in tubule formation. Angiogenesis 11: 153-167.

Farrell E, O'Brien F J, Doyle P, Fischer J, Yannas I, Harley BA, O'Connell B, Prendergast PJ, Campbell VA (2006) A collagen-glycosaminoglycan scaffold supports adult rat mesenchymal stem cell differentiation along osteogenic and chondrogenic routes. Tissue Eng 12: 459468.

Farrell E, Byrne EM, Fischer J, O’Brien FJ, O’Connell BC, Prendergast PJ, Campbell VA. (2007) A comparison of the osteogenic potential of adult rat mesenchymal stem cells cultured in 2-D and on 3-D collagen glycosaminoglycan scaffolds. Technol Health Care 5: 1931.

Farrell E, van der Jagt OP, Koevoet W, Kops N, van Manen CJ, Hellingman CA, Jahr H, O’Brien FJ, Verhaar JA, Weinans H, van Osch GJ (2009) Chondrogenic priming of human bone marrow stromal cells: a better route to bone repair? Tissue Eng Part C Methods 15: 285-295.

Finkenzeller G, Torio-Padron N, Momeni A, Mehlhorn AT, Stark GB (2007) In vitro angiogenesis properties of endothelial progenitor cells: a promising tool for vascularization of ex vivo engineered tissues. Tissue Eng 13: 1413-1420.

Hung SC, Pochampally RR, Chen SC, Hsu SC, Prockop DJ (2007) Angiogenic effects of human multipotent stromal cell conditioned medium activate the PI3K-Akt pathway in hypoxic endothelial cells to inhibit apoptosis, increase survival, and stimulate angiogenesis. Stem Cells 25: 2363-2370.

Ingber DE (2002) Mechanical signaling and the cellular response to extracellular matrix in angiogenesis and cardiovascular physiology. Circ Res 91: 877-887.

Jaasma MJ, O’Brien FJ (2008) Mechanical stimulation of osteoblasts using steady and dynamic fluid flow. Tissue Eng Part A 14: 1213-1223.

Jazayeri M, Allameh A, Soleimani M, Jazayeri SH, Piryaei A, Kazemnejad S (2008) Molecular and ultrastructural characterization of endothelial cells differentiated from human bone marrow mesenchymal stem cells. Cell Biol Int 32: 1183-1192.

Jukes JM, Both SK, Leusink A, Sterk LM, van Blitterswijk CA, de Boer J (2008) Endochondral bone tissue engineering using embryonic stem cells. Proc Natl Acad Sci USA 105: 6840-6845. 
Ko HC, Milthorpe BK, McFarland CD (2007) Engineering thick tissues - the vascularisation problem. Eur Cell Mater 14: 1-18.

Lee EJ, Niklason LE (2010) A novel flow bioreactor for in vitro microvascularization. Tissue Eng Part C Methods, in press.

Melero-Martin JM, Khan ZA, Picard A, Wu X, Paruchuri S, Bischoff J (2007) In vivo vasculogenic potential of human blood-derived endothelial progenitor cells. Blood 109: 4761-4768.

Melero-Martin JM, De Obaldia ME, Kang SY, Khan ZA, Yuan L, Oettgen P, Bischoff J (2008) Engineering robust and functional vascular networks in vivo with human adult and cord blood-derived progenitor cells. Circ Res 103: 194-202.

O’Brien FJ, Harley BA, Yannas IV, Gibson L (2004) Influence of freezing rate on pore structure in freeze-dried collagen-GAG scaffolds. Biomaterials 25: 1077-1086.

O’Brien FJ, Harley BA, Yannas IV, Gibson LJ (2005) The effect of pore size on cell adhesion in collagen-GAG scaffolds. Biomaterials 26: 433-441.

Oswald J, Boxberger S, Jorgensen B, Feldmann S, Ehninger G, Bornhauser M, Werner C (2004) Mesenchymal stem cells can be differentiated into endothelial cells in vitro. Stem Cells 22: 377-384.

Rouwkema J, Rivron NC, van Blitterswijk CA (2008) Vascularization in tissue engineering. Trends Biotechnol 26: 434-441.

Seghezzi G, Patel S, Ren CJ, Gualandris A, Pintucci G, Robbins ES, Shapiro RL, Galloway AC, Rifkin DB, Mignatti P (1998) Fibroblast growth factor-2 (FGF-2) induces vascular endothelial growth factor (VEGF) expression in the endothelial cells of forming capillaries: an autocrine mechanism contributing to angiogenesis. J Cell Biol 141: 1659-1673.

Simo R, Carrasco E, Garcia-Ramirez M, Hernandez C (2006) Angiogenic and antiangiogenic factors in proliferative diabetic retinopathy. Curr Diabetes Rev 2: 71-98.

Tierney CM, Haugh MG, Liedl J, Mulcahy F, Hayes B, O'Brien FJ (2009a) The effects of collagen concentration and crosslink density on the biological, structural and mechanical properties of collagen-GAG scaffolds for bone tissue engineering. J Mech Behav Biomed Mater 2: 202-209.

Tierney CM, Jaasma MJ, O’Brien FJ (2009b) Osteoblast activity on collagen-GAG scaffolds is affected by collagen and GAG concentrations. J Biomed Mater Res A 91: 92-101.

Ventura C, Cantoni S, Bianchi F, Lionetti V, Cavallini C, Scarlata I, Foroni L, Maioli M, Bonsi L, Alviano F, Fossati V, Bagnara GP, Pasquinelli G, Recchia FA, Perbellini A (2007) Hyaluronan mixed esters of butyric and retinoic Acid drive cardiac and endothelial fate in term placenta human mesenchymal stem cells and enhance cardiac repair in infarcted rat hearts. J Biol Chem 282 14243-14252.

Yamamura N, Sudo R, Ikeda M, Tanishita K (2007) Effects of the mechanical properties of collagen gel on the in vitro formation of microvessel networks by endothelial cells. Tissue Eng 13: 1443-1453.
Yannas IV, Lee E, Orgill DP, Skrabut EM, Murphy GF (1989) Synthesis and characterization of a model extracellular matrix that induces partial regeneration of adult mammalian skin. Proc Natl Acad Sci USA 86: 933937.

\section{Discussion with Reviewers}

Reviewer I: The authors have selected one particular type of scaffold structure for the study. Is this structure optimised for this particular application - or could further improvements potentially be made?

Authors: The scaffold used in this study was the 'standard' scaffold that we have used in our laboratory over the past number of years. It has a mean pore size of $96 \mu \mathrm{m}$, which has proved optimal for osteoblast adhesion (O'Brien et al., 2005, text reference; O'Brien et al., 2007). However, another recent study from our laboratory has shown that optimal pore size in these scaffolds is cell type specific (Byrne et al., 2008, text reference). Therefore, it is possible that the scaffold could be improved by analysis of the optimal pore size required for angio- and vasculogenesis and repeating the experiments in the current study with the modified scaffold. Recent modifications to our lyophilisation process now allow us to produce scaffolds with pares as large as $325 \mu \mathrm{m}$ (Haugh et al., 2010) and scaffolds with these larger pores have been shown to promote improved cell migration to the scaffold centre (Murphy et al., 2010). It is highly likely therefore, that scaffolds with larger pores would produce increased vasculogenesis, by simply providing more space for vessels to form. This will be considered in future experiments in our laboratory.

Reviewer II: As the authors point out themselves, there are well known shortcomings of the approach taken effectively precluding a conclusive outcome (for example lack of shear stress associated with vasculogenesis). Please comment!

Authors: We believe that this is an important study examining the suitability of MSCs to vascularise CG scaffolds in comparison to other vascular cells. One of the main outcomes of the study was that the data reveal that MSCs alone may not have the potential to form mature vascular cells in vitro and that further conditions may be necessary to promote this maturation. We conclude that ECs would be our candidate cell type for vascularisation moving forward. "ECs were also shown to form elaborate vascular-like structures within the CG scaffold and these may be the pro-angiogenic cell source of choice". We believe that this is an important outcome that will influence other researchers looking at candidate cell sources for vascularisation. We agree with the reviewer that the application of shear stress might have beneficial effect on vasculogenesis. Ongoing research in our laboratory is using a flow perfusion bioreactor to provide a biophysical environment to enhance osteogenesis and vasculogenesis (see for example: Jaasma et al. 2008; Jungreuthmayer et al. 2009; Plunkett et al., 2010). However, we feel that these types of experiments are beyond the scope of the current 
study and the inclusion of any bioreactor data would detract from the focus of the current paper.

Reviewer II: The authors base their paper on the premise that they are analysing mesenchymal stem cell differentiation into different cell types. However, based on the description of their procedure for cell isolation, these are effectively a mixture of adherent marrow cells. No doubt, there will some mesenchymal stem cells in this preparation. However, these may actually constitute only a small fraction of the total cell number. Without some stringent isolation/characterization these should not be labelled 'stem cells'. Cells were kept in monolayer culture in high glucose DMEM for a considerable number of passages prior to seeding onto the scaffolds. This is an important consideration as indicated by the data in Fig. 1, which clearly indicates phenotypic changes. This may not be related to differentiation but simply due to relative growth rates under these conditions and preferential enrichment of certain cell types in the population.

Authors: The MSCs isolation technique used in this study is a standard protocol published previously by the group (Farrell et al., 2006; Farrell et al., 2007; Byrne et al., 2008, text references). The cells isolated have been well characterized by both flow cytometry and their tri-lineage differentiation abilities. The techniques are in line with published work by other groups. There is a change in phenotype from P0-P3, which is part of the MSC isolation protocol. We added this information in for completeness sake to show that our culture techniques yielded a homogenous MSC population by P3, this was the cell passage used for all subsequent experiments described in the manuscript.

Reviewer II: How can the authors be sure that the 'network-like structures' in Fig. 4 are not simply a consequence of the cells adhering to the matrix scaffold? Not surprisingly in high serum/glucose DMEM the cells differentiate into myofibroblasts which contract the matrix scaffold. Therefore, the cells appear more densely and evenly distributed. If these network-like structures are tubes they should be 3-dimensional and this should be easy to check using confocal microscopy together with optically sectioning through the presumptive tube at different levels. It is pertinent to clearly demonstrate the relationship between cells and the scaffold to distinguish between cells associating with/growing around the scaffold and cells actually forming tube-like structures away from the scaffold.

Authors: The cells cultured in high/glucose DMEM caused considerable contraction of the scaffold (results depicted in Fig. 3), whereas the cells in EC media did not cause any contraction, this was also apparent in the endothelial cell only group, which did not cause contraction. We feel that this result highlights that the cells behave differently dependant on cell culture conditions, with MSCs under EC culture conditions behaving in a similar manner to endothelial cells. Furthermore, we have added new data to the manuscript (Fig. 9) to show that the tubules formed are caused by direct cell to cell interactions rather than cell-scaffold interaction. We trust that these new images provide more convincing evidence as to the 3 -dimensional nature of the structures and overcome the reviewer's concern about the lack of a conclusive outcome from the manuscript.

Reviewer II: The data analyzing expression of markers of endothelial cell differentiation is inconclusive (with the exception of PECAM there is no convincing upregulation of any other marker). The morphological data on formation of network-like structures is also not convincing by itself. Taken together, neither line of evidence supports the conclusion of tube formation by marrow cells sufficiently and therefore, the data is too preliminary to recommend publication.

Authors: We agree with this point and focus on this at the end of the discussion, where we draw the main conclusion that MSCs may not be the optimal cell source to vascularise the CG scaffold. However, we feel that in the morphological data set MSCs behave in a similar manner to ECs but do not adopt a full endothelial phenotype. As stated above, we think this is an important finding in this area of research and as described in the point immediately above, we trust that the inclusion of the new images provide more convincing evidence as to the 3-dimensional nature of the tubes formed.

\section{Additional References}

Haugh MG, Murphy CM, O’Brien FJ (2010) Novel freeze-drying methods to produce a range of collagenGAG scaffolds with tailored mean pores sizes. Tissue Eng Part C Methods, in press.

Jaasma MJ, O’Brien FJ (2008) Mechanical stimulation of osteoblasts by steady and dynamic fluid flow. Tissue Eng A 14: 1213-1223.

Jungreuthmayer C, Jaasma MJ, Al-Munajjed A, Zanghellini J, Kelly DJ, O'Brien FJ (2009) Deformation simulation of cells seeded on a collagen-GAG scaffold in a flow perfusion bioreactor using a sequential 3D CFDelastostatics model. Medical Eng Phys 31: 420-427.

Murphy CM, Haugh MG, O’Brien FJ (2010) The effect of mean pore size on cell attachment, proliferation and migration in collagen glycosaminoglycan scaffolds for tissue engineering. Biomaterials 31: 461-466.

O’Brien FJ, Harley BA, Waller MA, Yannas IV, Gibson LJ, Prendergast PJ (2007) The effect of pore size on permeability and cell attachment in collagen scaffolds for tissue engineering. Technol Health Care 15: 3-17.

Plunkett NA, Partap S, O’Brien FJ (2010) Osteoblast response to rest periods during bioreactor culture of collagen-glycosaminoglycan scaffolds. Tissue Eng A 16: 43-91. 